DOI: $10.3901 / J M E .2019 .08 .030$

\title{
覆膜铁覆膜理论与技术研究进展
}

\author{
张清东 刘吉阳 张勃洋 张立元 \\ (北京科技大学机械工程学院 北京 100083)
}

\begin{abstract}
摘要: 覆膜铁具有更好的节约资源能源和绿色环保安全的性能, 可替代镀锡板等包装用材, 是正在研发中的新型金属包装材 料。针对国内覆膜铁研发需求，从覆膜工艺、膜铁结合机理、覆膜质量评价与控制、基材表面预处理及膜表面改性等方面概 括梳理国内外关于覆膜铁覆膜理论与技术的研究进展, 指出膜与基材接触区温度场是决定界面连接乃至覆膜质量和速度的关 键, 实现覆膜区温度场稳定、控制界面空洞缺陷与膜内再结晶缺陷、减小横向与纵向膜厚波动是提高覆膜质量和效率的关 键; 提出从膜铁结合强度、膜厚均匀性、膜结晶率、塑性成形加工性能等不同角度对覆膜质量进行全面评价; 建议精细研 究接触区热行为和聚合物膜熔融及凝固过程, 从细观尺度研究熔融膜在基材粗徏表面受辊压作用流动、铺展、湿润、填充 行为, 从微观尺度研究膜铁化学反应键合与聚合物再结晶; 提出借鉴板形和厚度的控制理论与技术及其研究方法, 包括借 鉴轧机颤振问题研究考虑覆膜辊系动力学特性, 研究横向和纵向膜厚控制方法及技术。相关分析及建议有助于覆膜理论与 技术的创新研究。
\end{abstract}

关键词：覆膜铁；覆膜质量；熔融覆膜；金属包装

中图分类号: TF301

\section{Advances in Theory and Technology for Laminating of Polymer-coated Steel}

\author{
ZHANG Qingdong LIU Jiyang ZHANG Boyang ZHANG Liyuan \\ ( School of Mechanical Engineering, University of Science and Technology Beijing, Beijing 100083)
}

\begin{abstract}
Polymer-coated steel is a new type of metal packaging material under development, which has better performance in saving resources, energy, and environmental protection. It can replace packaging materials such as tinplate. In response to the research and development needs of domestic polymer-coated steel products, the research progress of the theory and technology of polymer-coated steel is analyzed and summarized. The trend of theory and technology development is studied from the laminating process, the combined mechanism of film and steel, the evaluation of laminating quality, the quality control of the film and the strip steel, the surface pretreatment and the surface modification of polymer film. It suggests that the temperature field of the contact zone between the film and steel is the key factor to determine the interface hydrogen bonding connection and even the film quality and film laminating speed. The temperature field stability of the film area and the controlling of interface cavity defect are studied. Intra-membrane recrystallization defects, reducing lateral and longitudinal film thickness fluctuations are the key to improving film quality and efficiency. We pointed out that the film quality should evaluated in different field such as bonding strength, film thickness uniformity, crystallization rate and plastic forming processing performance. The thermal behavior in the contact zone and the melting and solidification process of the polymer film should be studied. The flow, spreading, wetting and filling behaviors of the molten film under the action of roller on the rough surface of the substrate should be studied from the microscopic scale. The control theory and technology of the shape and thickness of the plate and the research methods be introduced, including the study of the dynamic characteristics of the coated roller system, and the lateral and longitudinal film thickness control methods and techniques are studied. In addition, several research proposals for improving film quality and efficiency are proposed, which may be helpful to the innovative research of current laminating theory and technology.
\end{abstract}

Key words: polymer-coated steel; laminating quality; thermal melting laminating; metal packaging

* 国家自然科学基金(U1760106)和中央高校基本科研业务费专项资金 (FRF-TP-17-010A1)资助项目。20181121 收到初稿, 20190115 收到修 改稿 


\section{0 前言}

物品包装及其原材料, 既要满足包覆保护的技 术功能要求, 也要迎合消费者对于美观性和安全性 的需要。因此, 包装材料的发展与材料制备及加工 技术、个人审美时尚以及社会的消费观念、发展观 念乃至文明意识等诸因素相关。覆膜铁, 正是因为 与现有的各类包装材料相比, 更能满足当今社会绿 色可持续发展要求, 能提供更好的安全性与个性化 消费体验, 且具有更高的包装技术性能, 从被发明 起一直受到金属包装行业的大力推崇, 并被认定是 将替代当前主流金属包装材料一一镀锡板(俗称马 口铁)的未来产品。

覆膜铁，一种金属与非金属层状复合带材 ${ }^{[1]}$, 替代包装用镀锡板能减少锡资源消耗, 避免制罐及 使用中污染问题，显著提高耐冲压、耐腐蚀、密闭 阻气性和热稳定性, 增加包装产品的美观性, 是低 成本、绿色环保、可回收的新型金属功能材料 ${ }^{[2-4]}$ 。

20 世纪 80 年代末, 日本东洋制罐公司率先研 发成功覆膜铁产品, 经过不断发展, 已在日本基本 实现覆膜铁替代马口铁, 并且形成相对完整的覆膜 铁生产和应用技术体系。1991 年, 欧美也投产覆膜 铁产品, 目前德、英、法、美等国均研制成功了能 够完全替代马口铁的覆膜铁, 但产量远不及日本 ${ }^{[5]}$ 。

我国覆膜铁研究起步较晚, 2004 年江苏无锡一 家企业首次制备出使用胶粘剂将聚合物薄膜和基板 秥结而成粘贴覆膜铁产品。粘贴覆膜铁的诞生引起 了国内对覆膜铁材料的关注, 2007 年宝钢建成了国 内最先进的镀铬带钢生产线, 拟为覆膜铁生产提供 基材带钢, 并开始寻求引进国外覆膜铁生产技术。 2010 年前后, 国内以宝钢、奥瑞金包装公司、中粮 集团为代表的传统金属包装厂商相继开始规划研发 熔融覆膜铁产品。奥瑞金公司于 2014 年宣布试验开 发成功熔融覆膜铁, 宝钢股份于 2016 年集成建设国 内第一条现代化覆膜铁生产线并生产出产品。与此 同时, 上海联净公司研制出了覆膜铁覆膜设备的核 心部件一一电磁感应加热辊, 进而研发了覆膜铁生 产技术并申请了专利 ${ }^{[6-7]}$ 。江苏无锡是国内最早的粘 贴覆膜铁的发源地, 目前该地许多民营企业也在生 产熔融覆膜铁。但是, 可用于食品饮料罐的熔融覆 膜铁, 由于其覆膜工艺参数窗口窄、覆膜过程稳定 控制难, 目前只有奥瑞金和宝钢能够生产，但是覆 膜质量、速度及生产稳定性等方面与国外先进水平 仍有较大差距。

在两种覆膜铁 ${ }^{[8]}$ 产品中, 粘贴覆膜铁由于其较
差的附着力一般只用于化工罐、装饰品等 ${ }^{[9-10]}$; 熔 融覆膜铁用于食品罐和饮料罐, 且因其具有与覆膜 铝相当的食品饮料风味保持能力和更环保、更低成 本而被寄希望于抢占取代覆膜铝独自垄断的啤酒罐 等最高端市场。覆膜铁常用的聚合物膜有聚对苯二 甲酸乙二醇酯 $(\mathrm{PET}) 、$ 聚乙烯 $(\mathrm{PE})$ 、聚丙烯 $(\mathrm{PP})$ 等, 而基材一般采用表面镀铬层厚度仅是传统镀锡板镀 锡层厚度几十分之一的镀铬板。一般认为, 覆膜铁 代表了食品包装材料的发展趋势 ${ }^{[11-13]}$ 。

\section{1 覆膜工艺与机理}

\section{1 覆膜工艺}

常见的金属表面覆膜或涂层方法有喷涂、电镀 和真空镀。其中，喷涂技术包括热喷涂技术和冷喷 涂技术 ${ }^{[14-16]}$ 。热喷涂是通过压缩气体将加热熔化的 金属或非金属材料连续吹喷到制件表面，形成与基 体牢固结合涂层的一种覆膜技术 ${ }^{[17-18]}$ 。冷喷涂技术 又称气体动力喷涂技术，是近十年来新兴的一种常 低温高速喷涂技术 ${ }^{[19-21]}$ 。覆膜铁覆膜技术与喷涂技 术类似，均是使用特定工艺将非金属材料涂覆于金 属材料表面。

针对覆膜铁产品, 其覆膜工艺技术也可以分为 三种：胶粘法覆膜技术、淋膜覆膜技术、热熔覆膜 技术 ${ }^{[22]}$ 。其中, 胶粘法覆膜是室温粘合覆膜, 其余 两种均为热覆膜。

胶粘法覆膜是由我国发明的一种覆膜技术，诞 生于国内覆膜铁行业的起步阶段。胶粘法覆膜是采 用胶粘剂将金属基板与聚脂薄膜黏合起来的一种覆 膜技术，覆膜过程采用室温黏合，常用的胶粘剂一 般为聚氨酯胶黏剂, 其钢板基板与聚脂薄膜的结合 强度与两种热覆膜方法相比有很大差距。目前随着 熔融覆膜技术的发展, 胶粘法覆膜也逐渐被取代, 不过仍有部分产品仍然采用胶粘法制备, 例如冰箱 表面贴膜等，该类产品覆膜的作用旨在提高材料美 观性，对膜铁结合质量并无太高要求。

热覆膜技术是继胶粘法覆膜技术之后出现的更 先进的覆膜技术, 可根据覆膜工艺分为淋膜覆膜技 术和热熔覆膜技术，也可根据覆膜设备区分为辊压 覆膜和静压覆膜。淋膜覆膜和热熔覆膜均可采用辊 压和静压方式, 但静压覆膜在实验室研究阶段就因 其不具备辊压覆膜的连续性优势而被放弃, 目前工 业生产中都使用辊压覆膜技术。

淋膜覆膜技术采用双层共挤方式将熔融树酯挤 压成膜直接贴合在金属基板上,进而冷却覆膜 ${ }^{[23-24]}$, 如图 1 所示。国内目前仅有少部分企业采用该覆膜 
技术, 该覆膜技术适用于单层薄膜的产品, 如化工 罐、气雾罐、装饰品等, 而不适合具有三层共挤结 构且外功能层可印刷商品图标 ${ }^{[25-27]}$ 的食品罐。但是, 淋膜覆膜技术将薄膜制备和覆膜过程结合为一体, 减少了薄膜的运输、分切等环节。

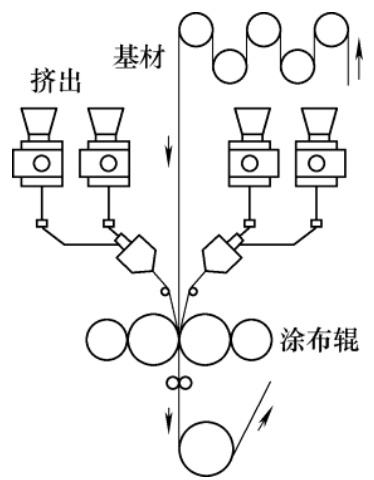

图 1 双层共挤涂膜系统示意图

热熔融覆膜工艺, 通过预热带钢基材并利用带 钢基材加热聚脂薄膜至其熔点以上使薄膜表层部分 熔化, 再在覆膜辊的辊压作用下使固态膜与基材之 间的熔融态聚合物在界面上充分流动、铺展、接触 以及冷却凝固, 直至膜与基材实现辊压复合, 进一 步冷却固化而成一种金属与非金属的层合带材 ${ }^{[28-29]}$, 如图 2 所示。

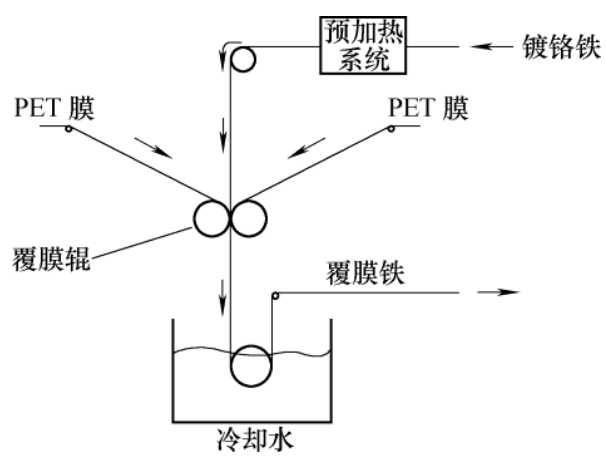

图 2 辊压热熔覆膜示意图

在辊压覆膜过程中, 膜铁接触界面处形成一种 带钢基材-熔融态聚合物-固态聚合物膜的三层结 构 ${ }^{[30-31]}$, 带钢基材和聚合物薄膜的温度精确控制与 覆膜辊压力分布均匀稳定是决定覆膜质量的关键所 在, 产品的膜隔离防护性能和膜铁结合力是其关键 质量指标。因此, 在生产过程中必须精确控制保证 的工艺参数包括覆膜辊压力、覆膜辊形变、覆膜速 度、基材温度、覆膜辊温度、薄膜温度、冷却水温 度及室温温度等。此外，基材表面预处理后的粗敉 度与镀铬层厚度, 以及聚合物膜表面改性预处理也 会影响覆膜质量。

\section{2 膜铁结合机理}

高分子材料在金属表面的粘附并相互结合的
现象是表面科学领域中的一个重要研究方向, 但 目前关于覆膜铁结合界面的粘附机理尚不完全清 楚。国内外比较公认的膜铁粘附机理认识主要有 四种: 机械啮合理论、静电理论、扩散理论、吸附 理论 ${ }^{[32-36]}$ 。

20 世纪 20 年代, MCBAIN 等 ${ }^{[37]}$ 提出了机械 啮合理论, 认为两种材料粘附现象是粘附材料与 基板不规则粗粘表面孔、缝隙等处形成机械联锁的 结果。

20 世纪 50 年代, DERYAGUIN 等 ${ }^{[38-40]}$ 提出静 电理论, 认为高分子材料和基板具有不同电子带结 构的情况下, 两材料在接触时可能发生电子的转移, 用来平衡费米能级，导致在高分子材料和基板结合 界面处形成了双层电荷, 通过接触或电位连接产生 的静电力为两材料界面粘附提供了附着力。静电理 论的提出在当时引起了许多争议, 部分学者认为静 电力为两材料粘附提供了粘附力, 但界面粘附力并 不是高分子材料和基板间的静电力，粘附力应同时 包含其他形式的相互作用力。随后，该理论由后面 提出的吸附理论得到了补充。

VOYUTSKII 等 ${ }^{[41-42]}$ 于 20 世纪 60 年代提出粘 附扩散理论, 认为高聚物对自身和彼此的内在粘附 是两种材料结合界面处聚合物的大分子或链段相互 融入和渗透的结果。该理论成立的前提条件是两种 材料的聚合物大分子或链段具有足够的流动性和相 似的溶解度。该理论适用于解释聚合物和热塑性塑 料之间的粘附。

吸附理论是目前被广泛认可的解释粘附现象 的理论, HUNTSBERGER 等 ${ }^{[33-46]}$ 对此理论进行了 深入研究。该理论认为, 两种材料在界面区域内实 现了足够密切的分子间接触时, 两种材料会因各自 表面原子间的相互作用力而实现粘附。

关于覆膜铁界面微观复合机制，目前国内外大 多数学者比较认同的是由 TANAKA 等于 1987 年提 出的氢键复合理论, 属于上述吸附理论中的一种, 即覆膜过程中, 聚合物薄膜中 $\mathrm{C}==0$ 的 $\mathrm{O}$ 与 TFS 表 面-OH 的 $\mathrm{H}$ 结合形成氢键 ${ }^{[47]}$ 。图 3 是 PET 与聚合 物薄膜联结的分子式 ${ }^{[5]}$ 。氢键的概念由 BERNAL

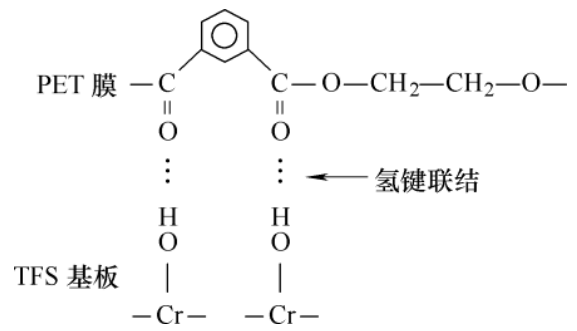

图 3 氢键联结分子式 
与 HUGGINS 在 1935 年正式提出 ${ }^{[48]}, \mathrm{KINLOCH}^{[32]}$, 张广宏等 ${ }^{[49]}$ 总结了原子间力类型和键能, 氢键的键能 在 $10 \sim 40 \mathrm{~kJ} / \mathrm{mol}$, 较范德华作用力(低于 $10 \mathrm{~kJ} / \mathrm{mol}$ ) 强, 但远小于通常离子键 $(590 \sim 1050 \mathrm{~kJ} / \mathrm{mol})$ 、共 价键 $(63 \sim 710 \mathrm{~kJ} / \mathrm{mol}) 、$ 金属键 $(113 \sim 347 \mathrm{~kJ} / \mathrm{mol})$ 强度。

从原子角度看, 有机高分子材料与金属基体之 间的微观空间就像是一个交换电子的场所, 有机高 分子材料中的含氧官能团、羟基、环氧化合物等作 为电子供体提供电子, 金属基板表面的金属离子接 收电子, 称为电子受体 ${ }^{[50-52]}$ 。1997 年日本学者駒井 正雄等 ${ }^{[53]}$ 指出覆膜铁微观界面内镀铬铁表面水合 氧化铬与聚合物薄膜间以形成氢键的形式进行联 结, 镀铬铁作为质子受体提供形成氢键所需的- $\mathrm{OH}$, 聚脂薄膜作为质子供体提供-O。上述研究指出覆膜 铁带钢基材与聚合物薄膜间以形成氢键的形式进行 连接, 但并未提及关于生成氢键的试验依据。这也 是目前覆膜铁微观界面粘附机理研究中所面临的问 题, 覆膜铁界面区域氢键复合理论的试验依据较少, 采用先进的试验设备与方法定量描述氢键生成工作 仍需进行。

ZUMELZU 等 ${ }^{[54]}$ 认为金属/聚合物界面复合机 制还可用另一种模型进行解释, 即酸碱相互作用机 制, PET 中羟基或者苯环中的氧原子与铬共用其电 子。LEWIS 等 ${ }^{[55]}$ 通过试验的方式采用非弹性隧道电 子显微镜研究了对苯二甲酸在金属铝表面的结构, 发现其构相为: 两个羧酸基之一与铝的氧化表面成 盐并垂直于表面, 另一个与相邻的羒酸以氢键相连。 CHTAIB 等 ${ }^{[56]}$ 利用 XPS 研究了金属铜与 PET 的界 面, 研究发现 PET 表面沉积的铜密度不高时, 铜原 子与 PET 形成了 $\mathrm{Cu}-\mathrm{O}-\mathrm{C}$ 的络合结构。该研究是采 用化学气相沉积的方法以 PET 为基材, 在 PET 表面 沉积铜原子进行的试验, 相比覆膜铁中聚合物与基 板表面水合氧化铬层的复合有一定的差异, 但可借 鉴该实验方法对覆膜铁内部熔融聚合物薄膜与水合 氧化铬层界面进行研究。

另外, 对于解释覆膜铁膜铁粘附结合机理具有 参考价值的是另一种金属与非金属层合带材一一彩 色涂层钢板(简称彩涂板)。它也是以金属带钢(冷轧 板、镀锌板、镀铝锌板)为基材, 通过在其表面涂覆 高分子涂料(聚酯、聚氨酯、环氧树脂)制备而成 ${ }^{[57-58]}$ 。 关于涂料与金属带钢界面结合机理也已提出许多学 说, 如机械咬合粘接理论、静电理论、吸附理论、 扩散理论、酸碱理论和化学理论等 ${ }^{[59-63]}$ 。路易斯酸 一碱相互作用力指的是 LEWIS 碱提供电子对的分 子、离子或原子团与能够接受电子对的 LEWIS 酸之
间的相互作用力。彩涂板中涂层提供电子对称为 LEWIS 碱, 基板接受电子对为 LEWIS 酸, 进而产 生相互作用力 ${ }^{[64]}$ 。

彩涂板和覆膜铁的界面结合机理有相似之处, 但两者实现结合的过程不同, 彩涂板在基板表面涂 覆有机涂料后再烘烤使涂料中溶剂挥发而溶质固化 于基板表面, 为提高涂层与基板结合强度需要对基 板表面进行化学转化处理。覆膜铁在基板和膜的固 固接触界面连续发生接触升温-表面熔融-辊压贴合氢键复合-冷却凝固等一系列物理化学反应, 从而实 现界面牢固结合而制成沿厚度方向材料分布为高聚 物薄膜-水合氧化铬-铬-钢板的层合板。

关于覆膜铁的界面结合机理, 目前尚未形成统 一的认识, 借鉴分子动力学、量子力学或者寻找新 的理论方法建立膜铁微观界面各基团间相互作用模 型以及探索膜铁微观界面粘附现象的试验表征新方 法都是值得深入研究的问题。

\section{3 覆膜过程热行为}

根据辊压熔融覆膜原理, 覆膜过程中聚合物薄 膜内部温度梯度的精确控制是覆膜铁能否成功覆膜 的关键。影响膜层内部温度的因素有：带钢基材温 度、聚合物薄膜温度、覆膜辊温度、室温温度 ${ }^{[22,26,53]}$ 。 国内外关于薄膜内部温度场变化规律的研究主要从 数理解析和试验研究两个角度展开。

数值解析是研究聚合物薄膜内部温度场变化规 律的重要研究手段。1987 年 TANAKA 等 ${ }^{[47]}$ 采用 SCHMIDT 解析法对 PET 薄膜内部温度变化规律进 行了计算, 如图 4 所示, 在带钢基材与 PET 薄膜接 触时, 薄膜贴近基材一侧发生部分熔化, 沿厚度方 向膜层内部温度逐渐降低, 随接触时长增加薄膜内 部温度逐渐趋于一致。该简易数理模型给出了膜层 内部的温度变化曲线, 为后续膜层温度场的研究提

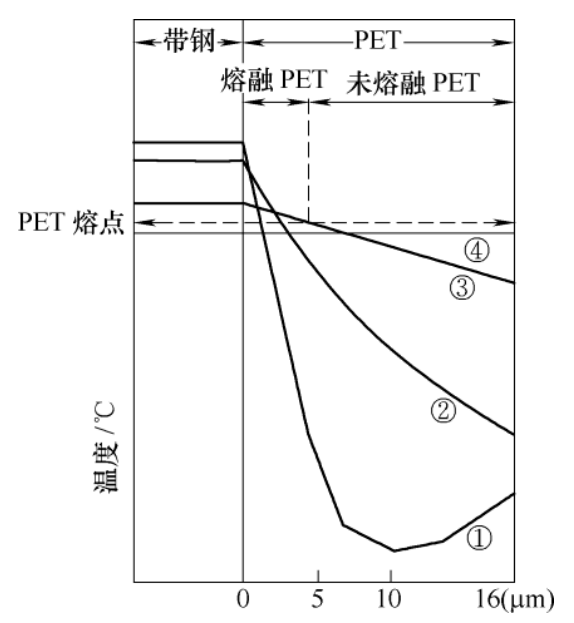

图 4 薄膜厚度方向温度分布示意图(1)为层压时; (2)为 $0.001 \mathrm{~s}$ 时; (3)为层压 $0.01 \mathrm{~s}$ 时; (4)为离开覆膜辊时) 
供了指导。2001 年日本学者岩下寛之 ${ }^{[65-66]}$ 使用一维 热传导方程式计算了不同初始覆膜辊温度、不同覆 膜速度下膜层内部温度变化规律, 并通过试验对计 算结果进行了验证。2015 年宝钢集团侯立刚等 ${ }^{[67]}$ 采用三维有限元法对覆膜过程中膜层内部温度场变 化规律进行建模, 精确计算出在薄膜与基板接触 $0.03 \mathrm{~s}$ 时, 薄膜内部接触覆膜辊侧表面温度达到最 高值, 随后薄膜内部温度逐渐趋于一致。

上述研究从解析分析到有限元法计算, 逐步精 确地研究膜内温度场变化规律, 边界条件虽逐渐增 多, 但均未考虑辊压力条件。覆膜过程中熔融聚合 物处于黏流体形态 ${ }^{[68-70]}$, 覆膜辊压力会对薄膜厚度 产生影响。从流变学角度, 熔融聚合物属于非牛顿 流体 ${ }^{[71-74]}$, 建立熔融态聚合物本构方程, 计算辊压 力条件下膜层内部温度变化规律, 建立精准的膜层 厚度预测模型是目前仍需深入的难题。

TANAKA 等 ${ }^{[47]}$ 研究了成品覆膜铁聚合物薄膜 中无定形层厚度对薄膜粘附性能和防腐性能的影 响, 试验结果表明, 随着无定形层厚度的增加薄膜 粘附性能增强, 同时无定形层厚度的增加导致了结 晶 PET 含量的减少, 进而造成了薄膜防腐蚀性能的 降低, 图 5、6 所示为随剩余 PET 含量增加薄膜水 透性和结合强度变化曲线。薄膜与基板附着力与复 合材料成型后薄膜耐蚀性相矛盾, 通过数据对比对 薄膜性能进行优化, 得出无定形层含量在 30\% $70 \%$, 覆膜铁综合性能最好。

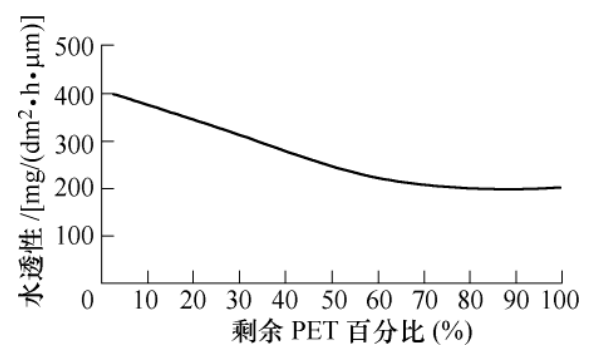

图 5 剩余 PET 百分比与水透性变化曲线

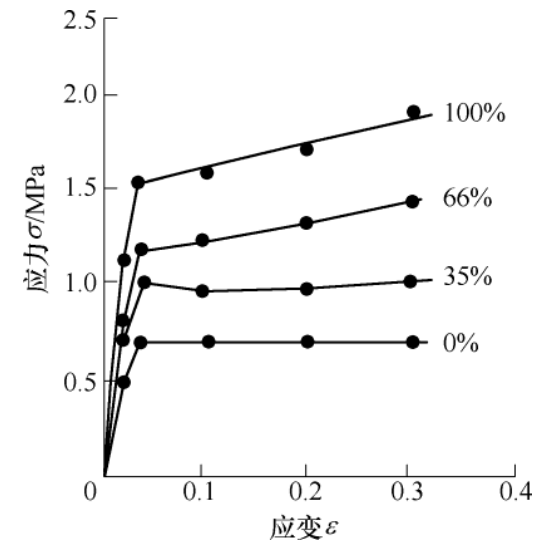

图 6 不同剩余 PET 百分比下薄膜应力-应变曲线
此后, 日本学者对覆膜铁开展了大量试验研究。 2000 年森田俊一等 ${ }^{[75]}$ 采用偏光显微镜和差示扫描 量热仪研究了不同层压温度对聚脂薄膜取向度的影 响, 而覆膜铁的许多特性都与聚脂薄膜取向度有关。 鈴木威等 ${ }^{[76]}$ 研究指出覆膜过程薄膜形变会降低膜 铁结合强度, 并提出通过尽可能减少无定型层的厚 度和控制基板表面镀铬层厚度及均匀性抑制薄膜形 变。TERAUCHI 等 ${ }^{[77]}$ 对分别使用 PET 和 PET/I 材料 制备的覆膜铁进行 50 240 ${ }^{\circ} \mathrm{C}$ 的热处理, 研究热处理 温度对结合强度的影响, 试验发现温度在 $160 \sim 180$ ${ }^{\circ} \mathrm{C}$ 时 PET/I 覆膜铁优于 PET 覆膜铁, 并认为是因 PET/I 覆膜铁的无定型层厚度大于 PET 覆膜铁所致。

基于更先进的试验仪器及设备, 1999 年韩国学 者 $\mathrm{CHO}$ 等 ${ }^{[78-79]}$ 通过 $\mathrm{X}$ 射线衍射、差式扫描量热 (DSC)、双折射试验研究了不同层压温度对薄膜性 能的影响。研究指出随着层压温度的升高薄膜无定 形层含量增加, 通过先进的试验方法和设备进一步 证实了 TANAKA 等的结论。针对工业生产需求, 2015 年丁丽萍等 ${ }^{[80]}$ 研制了一种用于覆膜铁生产的 高分子聚合物材料 PENIT, 并与 PET 膜对比研究, 发现使用 PENIT 的覆膜铁在相同基板温度下其结 晶度要高于 PET 覆膜铁, PENIT 材料结晶性能优于 PET。并且, 针对覆膜铁结合强度与防腐性能相矛 盾的约束, 优化覆膜工艺参数, 试验得出基板温度 在 $237{ }^{\circ} \mathrm{C}$ 时, 覆膜铁综合性能最好。

目前国内已初步掌握生产覆膜铁的辊压热熔覆 膜技术, 能够稳定批量生产但覆膜速度较低, 覆膜 质量与国外相比也有较大差距, 关于覆膜铁界面结 合机理的研究仍需进一步加强。此外, 认识和掌控 覆膜过程热行为是提高覆膜质量的重要抓手。覆膜 过程中基材温度、覆膜辊温度、薄膜温度、室温温 度共同决定了覆膜铁能否成功覆膜, 实现膜层的部 分熔化。膜层内部温度场的精确控制决定了膜层熔 融区厚度与未熔融区厚度, 熔融区厚度不同造成了 防腐蚀性能的不同, 进而影响覆膜铁覆膜质量。

\section{2 覆膜质量表征评价与控制}

覆膜铁覆膜质量的表征评价与控制是覆膜铁产 品生产和应用的关键。查阅文献资料从膜铁结合强 度与界面缺陷、膜厚均匀性、膜层结晶率与内部缺 陷、塑性成形加工性能等方面, 归纳和总结关于覆 膜质量评估与控制的研究进展。

\section{1 膜铁结合强度与界面缺陷}

覆膜钢板与薄膜间的结合强度和膜铁结合界面 间的真实复合面积是覆膜质量评估的重要指标。膜 
铁结合强度包括拉伸强度、剪切强度和剥离强度。 覆膜钢板和薄膜结合界面间的不完全结合状态用膜 铁真实复合面积表示。

针对膜铁结合强度, TANAKA 等 ${ }^{[47]}$ 通过试验指 出膜层与基板间剥离强度受膜层中无定形层厚度影 响, 无定形层越厚其剥离强度越高。岩下宽之等 ${ }^{[65]}$ 通过改变生产工艺, 增加覆膜过程中覆膜铁与覆膜 辊的接触弧长使膜铁粘附性能增强。分析原因认为 接触弧长的增加改变膜层内部温度场变化规律, 使膜层中无定形层厚度增加, 进而导致了粘附性 能的提高。丁丽萍课题小组研究了不同基板温度 对覆膜铁剥离强度的影响 ${ }^{[80]}$, 研究发现, 基板温度 在 $237{ }^{\circ} \mathrm{C}$ 之前, 覆膜铁剥离强度随温度升高线性增 加, 在 $237{ }^{\circ} \mathrm{C}$ 之后, 水合氧化铬与聚脂薄膜发生氢 键联结已经达到饱和, 覆膜铁剥离强度保持不变。

试验可见基板温度对膜铁结合强度影响很大。

膜铁界面缺陷指膜铁结合界面间的不完全结合 状态。熔融覆膜时, 薄膜被基板加热后贴近基板一 侧表层部分熔化, 随后薄膜与基板进行快速冷却使 熔化部分凝固, 完成覆膜铁的生产 ${ }^{[81]}$ 。而在覆膜过 程中在聚脂薄膜与基板接触时, 很有可能在基板与 薄膜中有空气渗入进而在薄膜内部产生气泡, 或者 薄膜在熔化后凝固过程中缩聚产生孔洞。气泡或孔 洞的产生, 再加上膜铁结合后重新断开等, 都可能 会导致在一定区域范围内薄膜与基板在界面层平面 内没有完全结合, 可用复合率即结合面积在总面积 中的占比表示。2004 年荷兰 CORUS 包装技术研发 中心的 BOELEN 等 ${ }^{[82-83]}$ 通过对 DRD (覆膜铁罐中的 一种)罐体进行盐水灭菌试验研究了覆膜铁罐体表 面小水泡类缺陷生成规律。ZUMELZU 等 ${ }^{[84-85]}$ 通过 扫描电镜试验也观测到了成型后聚脂薄膜表面有孔 洞存在。但其主题旨在研究基板表面镀铬层的特性, 对界面结合率并无提及。

膜铁结合强度既取决于膜与铁界面处生成氢键 的化学反应程度, 也与膜与铁结合界面的复合率密 切相关。因此, 研究探索提高膜铁结合强度的方法 及技术，需要同时研究影响氢键反应和复合率的因 素及其影响机制。此外, 膜铁结合强度与复合率的 实测表征方法及技术也是值得研究的课题。

\section{2 膜厚均匀性}

膜厚均匀性问题类似于板带轧制过程的厚度与 板形质量问题。成品覆膜铁的膜厚均匀性可区分为 膜层的横向厚度波动问题和纵向厚度波动问题。

目前国内关于覆膜铁膜厚均匀性方面的研究相 对较少, 并且主要是关于膜层结构与膜厚检测方面 的。辊压热熔复合膜层沿厚度方向有双层结构, 与
带钢基材结合的熔化后凝固的聚合物膜层(无定形 层)和原始聚合物膜层。2006 年 ZUMELZU 等 ${ }^{[54]}$ 使 用电子显微镜和红外光谱仪对覆膜铁界面层结构 进行了研究, 指出界面层结构依次为带钢基材-金 属铬层-水合氧化铬层-粘结层-无定形层-PET, 其中 镀铬层厚度和水合氧化铬层厚度均为 $0.01 \mu \mathrm{m}$, 粘结 层为 2 um。覆膜铁沿厚度方向界面层结构如图 7 所 示。聚合物膜厚可用在线测厚仪进行测量，或使用 立式光学仪离线测量, 其测量精度为 $0.1 \mu \mathrm{m}^{[86]}$ 。针 对薄膜内部无定形层厚度、可用扫描电镜、差式扫 描量热仪、 $X$ 射线衍射仪等设备直接或间接测量。

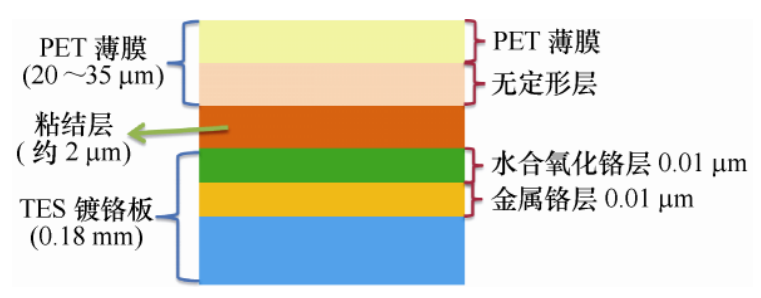

图 7 界面层结构示意图

根据板形生成与控制理论, 覆膜辊辊压力的横 向分布对膜层横向厚度均匀性起决定性作用, 而覆 膜辊的在线辊形(凸度与雉度)、两侧压下位置与轴 承跳动差、横向温度差、基材带钢的横向厚差、覆 膜辊弹性变形、辊系横刚度等诸多因素, 均会对辊 压力横向分布造成影响, 进而影响膜层的横向厚度 均匀性。这一方面完全可以参考板形研究的相关工 作 ${ }^{[87-92]}$ 开展类似研究。显然借鉴带钢轧制平整过程 板形横向厚差控制理论与技术研究方法, 建立覆膜 过程覆膜辊三维力学仿真有限元模型, 揭示膜层横 向厚度波动的机理与控制方法及技术。

根据厚度生成与控制理论, 覆膜辊辊缝开度的 纵向稳定性对膜层纵向厚度均匀性起决定性作用, 而覆膜辊的在线几何形状精度 (轴线偏心与不圆 度)、压下位置波动与轴承跳动、纵向温度波动、基 材带钢与聚合物膜的纵向厚度波动、覆膜辊系及机 架的弹性变形、机座纵刚度等诸多因素, 均会对覆 膜滚辊缝开度稳定性造成影响, 进而影响膜层的纵 向厚度均匀性。目前已经较为成熟的关于带钢厚度 控制理论与技术, 对于膜层纵向厚度均匀性控制具 有高度借鉴意义。当然, 由于覆膜过程中辊缝开度 与辊压力相互影响, 辊压力既影响膜层厚度也影响 界面结合强度与复合率, 因此膜层纵向厚度均匀性 控制将会与界面质量控制相互耦合。这可能是膜层 纵向厚度均匀性控制比板带轧制厚度控制更复杂的 方面。

此外, 关于覆膜铁界面层结构尤其是无定形层 与带钢基材间的粘结层的研究仍有待深入, 如何表 
征粘结层的物理化学性能、如何建模粘结层的形成 过程及影响因素等均需开展进一步的研究。

\section{3 膜层结晶率与内部缺陷}

覆膜铁聚合物薄膜对带钢基材的保护作用主要 体现在其具有耐热、耐腐蚀、防水、绝缘等性能。 聚合物薄膜内部无定形层厚度的不同导致了薄膜物

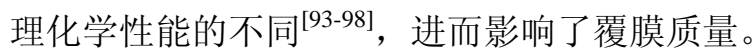

GAO 等 ${ }^{[99-102]}$ 通过试验的方法分别使用 $X$ 射线衍射仪、差示扫描量热仪、偏光显微镜检测 了聚合物材料的结晶度。借鉴上述试验方法对覆 膜铁进行薄膜结晶度测试时为满足试验设备制 样要求需将聚合物薄膜从带钢基材上 “无损剥

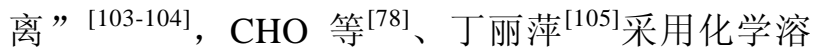
解法分别将成品覆膜铁置于 50\%体积浓度和 $30 \%$ 体积浓度盐酸溶液中, 将带钢基材溶解使膜铁分 离, 随后对薄膜进行蒸馏水冲和吹干从而获得完 整薄膜。ZUMELZU 等 ${ }^{[84]}$ 使用超薄切片术将 PET 从基材上切下, 获得成形后的 PET 薄膜。两种方 法均可满足薄膜结晶度的检测, 但试验步骤繁琐, 寻找新的覆膜铁薄膜结晶率试验检测方法及设备 仍是重要任务。

研究发现, 辊压热熔覆膜时, 膜层贴近基材一 侧因熔化转变为无定形物质, 后续冷却凝固且冷却 过程中会发生再结晶 ${ }^{[106-108]}$, 不过常用的聚合物材 料 PET 的再结晶速率极低, 较快的覆膜工艺冷却速 度使 PET 再结晶量可忽略不计 ${ }^{[109-111]}$, 此时可用无 定形层厚度表示膜层结晶行为。

$\mathrm{TANAKA}^{[47]} 、 \mathrm{CHO}^{[78]}$ 等先后通过试验制备了覆 膜过程不同基材温度下镀铬板和 PET 材料的覆膜铁 样板, 均使用差示扫描量热仪和 X 射线衍射仪对样 板进行了热性能测试和结晶率测试。试验指出, 随 着基材温度的升高 PET 薄膜内部无定形层厚度逐渐 增加、薄膜结晶率逐渐降低、粘附强度提高、薄膜 水透性提高、防腐蚀性降低, 决定薄膜水透性和耐 腐蚀性的是结晶层厚度, 而无定形层厚度的增加意 味着结晶层厚度减少。

丁丽萍等 ${ }^{[00]}$ 采用和 $\mathrm{CHO}$ 类似的试验方法得到, 在相同带钢初始预热温度下, 采用 PENIT 覆膜铁其 结晶度要高于 PET 覆膜铁, 成品覆膜铁薄膜结晶率 对薄膜性能有很大影响, 覆膜过程膜层内部温度场 变化是薄膜无定形层厚度的主要影响因素。同时, 使用 DSC 对薄膜进行热性能测试, 测量薄膜结晶区 域和非结晶区域不同的吸热放热规律, 使用如下公 式计算薄膜结晶率

$$
X_{\mathrm{c}}=\left(\Delta H_{\mathrm{m}}-\Delta H_{\mathrm{c}}\right) / \Delta H_{\mathrm{m}, \mathrm{o}} \times 100 \%
$$

式中, $X_{\mathrm{c}}$ 为聚合物薄膜结晶度, $\Delta H_{\mathrm{m}}$ 指薄膜的熔
解热, $\Delta H_{\mathrm{c}}$ 指薄膜的结晶热, $\Delta H_{\mathrm{m}, \mathrm{o}}$ 指薄膜在进行 覆膜前的熔解热。

该计算方法所得结果与 $X$ 射线衍射试验结果规 律一致, 但数值上有一定差距。

目前国内外关于薄膜结晶行为的研究已取得一 定成果, 结晶率与聚脂薄膜物理化学性能的研究已 较为丰富, 但关于覆膜铁覆膜过程中工艺参数对结 晶率影响的研究尚不够深入, 可进一步针对覆膜过 程中膜的热熔、流动、湿润、铺展、填充、凝固行 为对膜结晶行为的影响开展研究。

\section{4 膜层塑性成形加工性能}

覆膜铁的主要应用领域为金属包装工业 ${ }^{[112-115]}$, 因此制罐过程中覆膜铁材料的塑性加工性能也是评 价覆膜铁质量的重要指标。

目前国内针对覆膜铁产品的应用研究，主要集 中在覆膜铁冲压成形过程中膜层所出现的问题及其 解决对策方面 ${ }^{[116]}$ 。江轫等 ${ }^{[117]}$ 研究了冲压成形前热 处理对覆膜铁制罐白痕的影响, 发现较高的加热温 度和较长的保温时间促进了覆膜铁薄膜表面球晶的 转变, 在冲压成形时覆膜铁圆角处球晶在复杂应力 作用下发生破裂形成纤维结构，进而降低了薄膜透 明度形成白痕。倪俊义等 ${ }^{[118-119]}$ 提出了一种消除覆 膜铁制罐白痕缺陷的方法, 通过对 PET 薄膜进行加 热回复再结晶可使白痕处片晶和分子链取向重组形 成球晶结构, 进而白痕随取向消失而消除。

熔融覆膜质量与覆膜过程中膜层内部温度场密 切相关, 膜层厚向温度分布决定了成品覆膜铁薄膜 的结晶率, 结晶率的不同又导致了薄膜防腐蚀性能、 透水性能等的不同。

但是, 总体上, 目前国内外关于覆膜铁产品覆 膜质量的表征评估的方法与技术仍需要进一步研究 完善, 建立统一的、科学的覆膜铁覆膜质量量化评 定体系是迫切需求，关于覆膜质量控制理论与技术 的研究可以参考板带轧制过程的厚度与板形控制全 面展开。

\section{3 覆膜基材表面预处理技术}

覆膜铁的基材为镀铬铁, 目前国内已可稳定批 量生产，但对于低制耳(材料各向同性)基材尤其是 厚度小于 $0.15 \mathrm{~mm}$ 甚至更薄的基材尚还在努力研究 开发中。

镀铬铁一般采用水合电解铬的方式在电解质溶 液中于带钢基材表面电镀一层厚度 $50 \sim 150 \mathrm{mg} / \mathrm{m}^{2}$ 的金属铬层, 同时金属铬层与电解液反应生成厚度 约为 $50 \mathrm{mg} / \mathrm{m}^{2}$ 以内的水合氧化铬层, 形成一种基 
板-铬-水合氧化铬的三层结构 ${ }^{[22]}$, 如图 7 所示。镀 铬前需对钢板表面进行脱脂处理 ${ }^{[120-121]}$ 。

国内刘志浩 ${ }^{[22]}$ 对国外各镀铬板厂商生产的 TFS 板进行了统计, CORUS、ARCELORMITTLA、 RASSELSTEIN 三家公司生产镀铬板中金属铬层厚 度均为 $50 \sim 140 \mathrm{mg} / \mathrm{m}^{2}$ 、铬水合氧化铬层厚度约 7 $35 \mathrm{mg} / \mathrm{m}^{2}$, 而日本作为覆膜铁的发源地, 日本钢铁 工程控股公司 JFE 用于食品罐的覆膜铁的金属铬层 厚为 $120 \mathrm{mg} / \mathrm{m}^{2}$ 、水合氧化铬厚度 $15 \mathrm{mg} / \mathrm{m}^{2}$ 。

带钢基材表面形貌对覆膜铁粘附强度的影响尤 其显著。OCHOA-PUTMAN 等 ${ }^{[122]}$ 制备了不同表面 粗䊁度的带钢基材, 使用 PP 薄膜进行覆膜试验并 检测粘附强度。试验结果指出随着基板表面粗粘度 的逐渐增加, 样板剥离强度逐渐升高, 但当粗䊁度 增加到一定程度时粘附强度将不再增加。駒井正雄 等 ${ }^{[53]}$ 通过试验发现镀铬板表面水合氧化铬层增厚 时, 覆膜铁的密封性存在一个先变好后变差的过程, 分析原因认为当镀铬板表面水合氧化铬层增厚时镀 铬板与聚脂薄膜间的真实接触面积将增大, 有益于 提高聚脂薄膜的附着力, 但同时随着水合氧化铬层 厚度的增加, 基板表面微观形貌起伏增大, 引起较 高黏度的 PET 分子难以深入谷底或者由于谷底储存 的杂质劣化了膜与基板的综合性能。

带钢基材表面预处理在一定程度上可提高膜铁 结合强度, 改善覆膜铁覆膜质量。开展基材表面微 观形貌与膜铁粘附强度、膜铁界面结合率间的相互 影响关系研究, 探索改善覆膜质量的基材表面预处 理新方法依然是非常重要且必要的研究方向。

\section{4 膜表面改性技术}

覆膜铁用聚合物膜常用的表面改性处理方法 有: 电晕处理 ${ }^{[123]}$ 、等离子体处理 ${ }^{[124]}$ 、火焰处理 ${ }^{[125]}$ 、 表面接枝改性 ${ }^{[126]}$ 、离子注入表面改性 ${ }^{[127]}$ 等。其中, 电晕处理和等离子体处理是覆膜铁中比较常用的两 种表面改性手段。

电晕处理对聚酯薄膜表面结构和性能的影响主 要体现在薄膜物理化学结构、表面形态、表面能等 方面 ${ }^{[128-130]}$ 。电晕处理薄膜提高其表面能, 增加与 基材的附着力。电晕处理相较于其他处理, 耗时短、 速度快, 但效果不稳定, 表面能退化严重, 使用电 晕处理后应及时进行印刷、复合和粘接 ${ }^{[131-132]}$ 。

CARRADÒ 等 ${ }^{[133]}$ 通过试验发现覆膜前对薄膜 进行 $120 \mathrm{~s}$ 电晕处理可提高成型后材料力学性能, 同时指出聚酯薄膜表面极性官能团的增加提高了覆 膜铁粘附强度。BARBARO 等 ${ }^{[134]}$ 研究了电晕处理对
膜铁材料薄膜黏附性能的影响, 试验发现电晕处理 时间的增加和强度的提高增加了 PET 和基材见的黏 附强度。GIOVANNI 认为电晕处理提高材料粘附性 能的本质是电晕处理使薄膜表面引入了极性基团增 加了薄膜表面能, 改善了 PET 在基材上的铺展性。 NANTICHA 等 ${ }^{[135-137]}$ 通过试验说明了电晕处理效果 可持续时间短, 电晕效果逐渐消失，通过试验发现 电晕处理一段时间后薄膜表面基团发生了重排与迁 移，而基团的迁移正是导致该结果的主要原因。

等离子体处理技术也可对聚合物薄膜进行改性 处理, 提高聚合物薄膜粘附性和湿润性。韩国延世 大学 HAN 等 ${ }^{[138]}$ 通过试验研究了等离子体处理对覆 膜铁黏附性能的影响, 研究指出带钢基材表面的等 离子体处理使覆膜铁粘附强度增加, 且两者之间呈 线性正相关，认为等离子体处理增加了钢板基板表 面氧化程度, 增加了亲水性集团。PELAGADE 等 ${ }^{[139]}$ 利用 $\mathrm{X}$ 射线光电子能谱仪、原子力显微镜研究了等 离子体处理对 PET 表面自由能的影响, 研究指出随 着等离子体处理时间的增加, 其表面 $\mathrm{C}-\mathrm{O}$ 和 $\mathrm{C}=\mathrm{O}$ 基团增加, PET 薄膜表面水接触角由 $60^{\circ}$ 降低 到 $15^{\circ}$ ，表面自由能增加。

此外, PET 薄膜改性方式还有共混改性 ${ }^{[140]}$ 、化 学改性 ${ }^{[141]}$ 、纳米无机物改性 ${ }^{[142]}$ 等, 目的都是为了 提高 PET 薄膜粘附强度。

综上，对聚合物薄膜改性处理旨在提高其表面 极性集团含量增加其粘附强度。目前我国的覆膜用 聚合物薄膜产品种类及性能与国外比仍有一定差 距, 需要从保证覆膜质量和提高聚合物薄膜的耐深 冲性与防腐蚀性方面进一步开展研究。

\section{5 结论与展望}

金属包装材料的国内外市场需求巨大而且还在 不断增长中，全社会的可持续资源使用与绿色环保 发展理念以及消费者的个性化美观宜人要求，是当 前金属包装材产品发展面临的重大挑战, 覆膜铁应 运而生具有满足这一发展趋势及需求的潜力。然而, 我国的覆膜铁产品尚在成长发展中，虽已建设投产 了多条覆膜铁生产线, 但产品的品种与档次都不及 国外先进水平, 产品的覆膜质量(包括结合强度、复 合率、膜结晶度)有一定差距, 生产效率(尤其覆膜 工艺速度、成材率)也有明显差距。

我国覆膜铁产品差距的产生根源在于我国针对 覆膜铁覆膜理论与技术研究存在较大差距, 我国现 有主要生产线都是模仿、借鉴或者引进国外的关键 工艺与装备技术而建成的, 尚有若干重要覆膜理论 
与技术问题需要开展研究。

（1）覆膜过程的热行为。包括基材带钢、聚合 物膜、覆膜辊的覆膜全过程的精确温度场及其受控 变化规律, 聚合物膜的受控融化过程及熔融量, 熔 融聚合物的受控凝固过程及成膜层厚。

（2）熔融聚合物的粗糙表面流动行为。包括聚 合物膜表层熔融后在复合界面间受辊压力作用的流 动、铺展、湿润、填充以及基板表面微观形貌对于 这一过程的影响, 空气在复合界面间的卷入与挤出 和界面间气泡的生成, 基板表面微观形貌、辊压力、 覆膜辊转速与表面压扁变形对于界面结合强度、复 合率、气泡的影响。

(3) 膜铁化学反应键合与聚合物结晶。包括试 验表征描述微观界面氢键生成过程, 结合分子动力 学、量子力学等方法解释覆膜铁微观界面各基团间 相互作用关系，各项工艺温度及辊压力与辊压时间 对于氢键生成的影响, 冷却工艺、辊压力及辊压区 长度对于聚合物结晶过程的影响。

(4) 覆膜辊辊压力横向分布与横向膜厚均匀 性。在深入研究认识辊压覆膜与板带轧制在轧件方 面的显著不同的基础上，借鉴板形(横向厚差)控制 理论与技术以及板形(横向厚差)研究方法, 开展熔 融覆膜过程辊压力横向分布规律、横向膜厚波动规 律以及横向膜厚均匀性控制方法与技术。

(5) 覆膜辊辊缝开度稳定性与纵向膜厚均匀性。 借鉴厚度控制理论与技术以及厚度研究方法, 开展 熔融覆膜过程辊缝开度与辊压力波动规律、纵向膜 厚波动规律以及纵向膜厚均匀性控制方法与技术。

(6) 覆膜辊系的旋转运动与动力学特性及其对 于辊缝开度、辊压力、膜厚均匀性的影响。借鉴板 带轧机振动问题研究的方法与成果, 开展覆膜辊的 旋转及振动对于辊缝开度、辊压力、膜厚均匀性的 影响以及解决对策。

\section{参 考 文 献}

[1] 陈云鹏, 黄久贵, 李建中, 等. 覆膜铁的产品特性及其 国内外发展状况[J]. 轧钢，2010，27(1)：45-47.

CHEN Yunpeng, HUANG Jiugui, LI Jianzhong, et al. Properties of laminated steel product and its development situation[J]. Steel Rolling, 2010，27(1): 45-47.

[2] 杨文亮. 包装用马口铁的应用与发展 [J]. 中国包装, 1998(2): 51-52.

YANG Wenliang. Application and prospects of tinplate for packaging[J]. China Packaging, 1998(2): 51-52.

[3] 夏利勇, 邓潮玉, 齐永杰. 覆膜铁技术在我国的发展前 景预测 [J]．中国包装工业，2013(4)：12.
XIA Liyong, DENG Chaoyu, QI Yongjie. Prediction of the development prospect of coated iron technology in China[J]. China Packaging Industry, 2013(4): 12.

[4] 刘连喜, 刘建兵. 中国金属包装产业及包装材料的发展 现状及趋势[J]．河北冶金，2015(7)：76-79.

LIU Lianxi, LIU Jianbing. Development situation and trend of metal package industry and package materials at home[J]. Hebei Metallurgy, 2015(7): 76-79.

[5] 张清, 郭文渊, 丁树民, 等. 我国覆膜铁新材料的研究 与应用[J]. 上海包装, 2017(4)：58-61.

ZHANG Qing, GUO Wenyuan, DING Shumin, et al. Research and application of new polymer coated steel materials in China[J]. Shanghai Packaging, 2017(4): $58-61$.

[6] 文元庆, 邹斌. 一种覆膜铁加工工艺及设备, $\mathrm{CN}$ 102431269B[P]. 2012-05-02.

WEN Yuanqing, ZOU Bin. A processing technology and equipment for ploymer coated steel, CN 102431269B[P]. 2012-05-02.

[7] 文元庆, 邹斌. BOPET 薄膜与无锡钢板的热复合工艺及 设备, CN102514347A[P]. 2012.

WEN Yuanqing, ZOU Bin. Thermal composite technology and equipment of BOPET film and wuxi steel plate, CN102514347A[P]. 2012

[8] 唐卫. 覆膜铁如何尽快走出实验室实现产业化?[J]. 中 国包装工业，2013(B12)：30-33.

TANG Wei. How can the ploymer coated steel get out of the laboratory as soon as possible to achieve industrialization?[J]. China Packaging Industry , 2013(B12): 30-33.

[9] 肖艳. 发展中的中国食品金属包装工业及其市场前景 [J]. 饮料工业，2013，16(5)：2-8.

XIAO Yan. Development and market prospects of Chinese metal packaging industry for food $[\mathrm{J}]$. The Beverage Industry, 2013(B12): 30-33.

[10] 高康. 覆膜铁在金属包装上的应用 $[\mathrm{J}]$. 上海包装, 2016(10): 59-61.

GAO Kang. Application of ploymer coated steel on metal packaging[J]. Shanghai Packaging, 2016(10): 59-61.

[11] 李进卫. 覆膜铁金属容器包装材料及其应用技术 $[\mathrm{J}]$. 湖南包装，2014(4)：29-34.

LI Jinwei. Ploymer coated steel container packaging material and its application technology[J]. Hunan Packaging, 2014(4): 29-34.

[12] 杨文亮. 新型金属包装材料涂层钢板及其发展 $[\mathrm{J}]$. 上 海包装，2006(2)：32-33.

YANG Wenliang. New metal packaging material coating steel and its development[J]. Shanghai Packaging, 2006(2): 32-33. 
[13] 宋伟伟, 王洁琼, 柏建国, 等. 覆膜铁与涂料铁在金属 包装应用中的性能研究[J]. 中国包装工业, 2015(21): 46-48.

SONG Weiwei, WANG Jieqiong, BAI Jianguo, et al. Research on properties of ploymer coated steel and coating iron in metal packaging applications[J]. China Packaging Industry, 2015(21): 46-48.

[14] GÄRTNER F, STOLTENHOFF T, SCHMIDT T, et al. The cold spray process and its potential for industrial applications[J]. Journal of Thermal Spray Technology, 2006, 15(2): 223-232.

[15] GÄRTNER F, STOLTENHOFF T, VOYER J, et al. Mechanical properties of cold-sprayed and thermally sprayed copper coatings[J]. Surface and Coatings Technology, 2006, 200(24): 6770-6782.

[16] MORGAN R, FOX P, PATTISON J, et al. Analysis of cold gas dynamically sprayed aluminium deposits[J]. Materials letters, 2004, 58(7-8): 1317-1320.

[17] KUMAR S, BAE G, LEE C. Influence of substrate roughness on bonding mechanism in cold spray[J]. Surface and Coatings Technology, 2016， 304: 592-605.

[18] 陈书赢, 王海斗, 徐滨士, 等. 热喷涂层滚动接触疲劳 寿命演变规律研究进展 [J]. 机械工程学报, 2014, 50(8): 23-33.

CHEN Shuying, WANG Haidou, XU Binshi, et al. Law of rolling contact fatigue life of thermal spray coatings: a review[J]. Journal of Mechanical Engineering, 2014, 50(8): 23-33.

[19] ZAHIRI S H, ANTONIO C I, JAHEDI M. Elimination of porosity in directly fabricated titanium via cold gas dynamic spraying[J]. Journal of Materials Processing Technology, 2009, 209(2): 922-929.

[20] MOY C K S, CAIRNEY J, RANZI G, et al. Investigating the microstructure and composition of cold gas-dynamic spray (CGDS) Ti powder deposited on Al 6063 substrate[J]. Surface and Coatings Technology, 2010, 204(23): 3739-3749.

[21] XIE Yingchun, YIN Shuo, CHEN Chaoyue, et al. New insights into the coating/substrate interfacial bonding mechanism in cold spray[J]. Scripta Materialia, 2016, 125: $1-4$.

[22] 刘志浩, 曾科, 赵宇晖. 覆膜铁: 金属食品包装的“绿 色革命”[J]. 包装学报，2012(4)：9-15.

LIU Zhihao, ZENG Ke, ZHAO Yuhui. Polymer-coated steel: a green revolution in metal packaging for food and beverage[J]. Packaging Journal，2012(4): 9-15.

[23] 陈耀渠, 杨伟光. "挤出淋膜法"金属卷材复膜技术创新 一一智能数控共挤流涎复膜装备的诞生 [J]. 塑料包装, 2015, 25(6): 36-40.
CHEN Yaoqu, YANG Weiguang. Extrusion coating and laminanting method, the innovation of lamination technology for metal coils - the birth of intelligent CNC co-extrusion casting equipments[J]. Plastics Packaging, 2015, 25(6): 36-40.

[24] 厉善君, 万善红. 一种淋膜机上的内层膜放卷装置, CN204957888U[P]. 2016-01-13.

LI Shanjun, LI Shanhong. An inner membrane rewinding device on a film drainage machine, CN204957888U[P]. 2016-01-13.

[25] 苏志钢, 赵富, 田立斌, 等. 用于覆膜铁的可直接复合 的低熔点聚酯薄膜的制备方法, CN103753932A[P]. 2014-04-30.

SU Zhigang, ZHAO Fu, TIAN Libin, et al. Method for preparing direct composite low melting point polyester film coated with iron, CN103753932A[P]. 2014-04-30.

[26] 白永平, 丁丽萍, 邵路. 一种三层共挤耐高温蒸煮双向 拉伸聚酯薄膜, CN103112230A[P]. 2013-05-22.

BAI Yongping, DING Liping, SHAO Lu. A three-layer co-extrusion high temperature resistant cooked bidirectional tensile polyester film, CN103112230A[P]. 2013-05-22.

[27] 钟玉, 王慧荣, 董子尧. 一种热压熔融式覆铁膜, CN203844354U[P]. 2014-09-24.

ZHONG Yu, WANG Huirong, DONG Ziyao. Hot pressed molten iron coated film, CN203844354U[P]. 2014-09-24.

[28] 傅晓华. 覆膜铁产品及其制罐技术的研究 [J]. 上海包 装, 2013(4): 40-43.

FU Xiaohua. Research on polymer coated steel products and their can making technology[J]. Shanghai Packaging, 2013(4): 40-43.

[29] PALKOWSKI H, LANGE G. Production and forming behaviour of austenitic steel sandwich composites with a polymer core layer[J]. Materialwissenschaft und Werkstofftechnik, 2007, 38(2): 85-89.

[30] 乔军. 无锡钢在我国的应用及发展 $[\mathrm{J}]$. 中国冶金, 2000(6): 12-13.

QIAO Jun. Application and development of Wuxi Steel in China[J]. China Metallurgy, 2000(6): 12-13.

[31] ZUMELZU E, ANGULO C, CABEZAS C, ET AL. Characterisation of nanometric chromium coatings in metalâ polymer composites[J]. Surface Engineering, 2013, 29(8): 620-626.

[32] KINLOCH A J. The science of adhesion[J]. Journal of Materials Science, 1980, 15(9): 2141-2166.

[33] WAKE W C. Adhesion and the formulation of adhesives[M]. Barking: Elsevier Science Publishing, 1982.

[34] FOURCHE G. An overview of the basic aspects of 
polymer adhesion. Part I: Fundamentals[J]. Polymer Engineering \& Science, 1995, 35(12): 957-967.

[35] SEMOTO T, TSUJI Y, YOSHIZAWA K. Molecular understanding of the adhesive force between a metal oxide surface and an epoxy resin[J]. The Journal of Physical Chemistry C, 2011, 115(23): 11701-11708.

[36] SEMOTO T, TSUJI Y, YOSHIZAWA K. Molecular understanding of the adhesive force between a metal oxide surface and an epoxy resin: Effects of surface water[J]. Bulletin of the Chemical Society of Japan, 2012, 85(6): 672-678.

[37] MCBAIN J W, HOPKINS D G. On adhesives and adhesive action[J]. The Journal of Physical Chemistry, 1925, 29(2): 188-204.

[38] DERJAGUIN B V. Problems of adhesion[J]. Progress in Surface Science, 1994, 45(1-4): 223-231.

[39] DERJAGUIN B V, KROTOVA N A, KARASSEV V V, et al. Electrical phenomena accompanying the formation of new surfaces, and their role in adhesion and cohesion[J]. Progress in Surface Science, 1994, 45(1-4): 95-104.

[40] DERJAGUIN B V. Mechanical properties of the boundary lubrication layer[J]. Progress in Surface Science, 1994, 45(1-4): 328-336.

[41] VOYUTSKII S S, USTINOVA Z M. Role of autohesion during film formation from the latex[J]. The Journal of Adhesion, 1977, 9(1): 39-50.

[42] VOYUTSKII S S. Some comments on the series of papers "interfacial contact and bonding in autohesion" [J]. The Journal of Adhesion, 1971，3(1): 69-76.

[43] HUNTSBERGER J R. The nature of adhesion[J]. Adhesives Age, 1970, 13(11): 43.

[44] POSSART W. Experimental and theoretical description of the electrostatic component of adhesion at polymer/metal contacts[J]. International Journal of adhesion and adhesives, 1988, 8(2): 77-83.

[45] FOURCHE G. An overview of the basic aspects of polymer adhesion. Part I: Fundamentals[J]. Polymer Engineering \& Science, 1995, 35(12): 957-967.

[46] FOURCHE G. An overview of the basic aspects of polymer adhesion. Part II : Application to surface treatments[J]. Polymer Engineering \& Science, 1995, 35(12): 968-975.

[47] TANAKA A, HANAFUSA T, KOJYO H, et al. Adhesion of Biaxially Oriented Polyethylene Terephthalate Film to Tin Free Steel[J]. Transactions of the Iron \& Steel Institute of Japan, 2009， 27(8): 638-644.

[48] SCHÖNHERR H, PARASCHIV V, ZAPOTOCZNY S, et al. Supramolecular chemistry and self-assembly special feature: unraveling the nanostructure of supramolecular assemblies ofhydrogen-bonded rosettes on graphite: An atomic force microscopystudy $[\mathrm{J}]$. Proceedings of the National Academy of Sciences of the United States of America, 2002, 99(8): 5024-5027.

[49] 张广宏. 氢键的形成条件及对物质性质的影响 [J]. 宁 夏师范学院学报，2007，28(3)：84-88.

ZHANG Guanghong. Hydrogen bond formation conditions and effects on material properties[J]. Journal of Ningxia Normal University, 2007, 28(3): 84-88.

[50] CROSSLAND J L, TYLER D R. Iron-dinitrogen coordination chemistry : Dinitrogen activation and reactivity[J]. Coordination Chemistry Reviews, 2010, 254(17-18): 1883-1894.

[51] DERJAGUIN B V, SMILGA V P. Electronic theory of adhesion[J]. Journal of applied Physics, 1967, 38(12) : 4609-4616.

[52] VON HARRACH H G, CHAPMAN B N. Charge effects in thin film adhesion[J]. Thin Solid Films, 1972, 13(1): $157-161$.

[53] 駒井正雄, 谷口歩, 清水信義, 等. ラミネート鋼板の フィルム密着性におょぼす TFS のクロム水和酸化 物の組成と表面形態の影響 $[\mathrm{J}]$. 鐵と鋼 : 日本鐵鋼協 會々誌, 1997, 83(6): 377-382.

KOMAI M, TANIGUCHI A, SHIMIZU N, et al. Effects of composition and microstructure of hydrated chromium oxide on adhesive property of PET/I film laminated TFS[J]. Tetsu-to-Hagane, 1997, 83(6): 377-382.

[54] ZUMELZU E, RULL F, SCHMIDT P, et al. Structural analysis of polymer-metal laminates by electron microscopy and infrared spectroscopy[J]. Surface Coatings International Part B: Coatings Transactions, 2006, 89(1): 57-62.

[55] LEWIS D M, FIELD B O. Inelastic electron tunnelling spectroscopy-III. A study of the molecular orientation of phthalic, isophthalic and terephthalic acids adsorbed on alumina[J]. Spectrochimica Acta Part A Molecular Spectroscopy, 1985, 41(3): 477-481.

[56] CHTAIB M, GHIJSEN J, PIREAUX J J, et al. Photoemission study of the copper/poly(ethylene terephthalate) interface.[J]. Phys Rev B Condens Matter, 1991, 44(19): 10815-10825.

[57] 明振涛, 黄大华, 侯广亚, 等. 热镀锌彩涂板的失效与 防护[J]. 材料保护, 2015, 48(11): 43-47.

MING Zhentao, HUANG Dahua, HOU Guangya, et al. Failure and protection of hot-dip galvanized color coated sheets[J]. Materials Protection, 2015, 48(11): 43-47.

[58] 赵金榜. 彩涂板、彩涂板涂料和工艺的现状及其发展 [J]. 现代涂料与涂装，2006，9(1)：43-46. 
ZHAO Jinbang. Present situation and development of color coated metals, color coated metal coating and process theirof[J]. Modern Paint \& Finishing, 2006, 9(1): 43-46.

[59] 王雷. 彩涂板涂层附着力时效性研究[D]. 沈阳: 沈阳理 工大学, 2009 .

WANG Lei. A study on the lifespan of pre-coating sheet adhesion[D]. Shenyang: Shenyang Ligong University, 2009.

[60] LEE H Y, QU J. Microstructure, adhesion strength and failure path at a polymer/roughened metal interface[J]. Journal of Adhesion Science and Technology, 2003, 17(2): 195-215.

[61] LEE H Y, KIM Y H, CHANG Y K. Fracture behaviors of nanowire-coated metal/polymer systems under mode-I loading condition[J]. Acta Materialia, 2004, 52(20): 5815-5828.

[62] MCCAFFERTY E. Acid-base effects in polymer adhesion at metal surfaces[J]. Journal of Adhesion Science \& Technology, 2002, 16(3): 239-255.

[63] TAHARA M, CUONG N K, NAKASHIMA Y. Improvement in adhesion of polyethylene by glow-discharge plasma[J]. Surface \& Coatings Technology, 2003, 174(3): 826-830.

[64] 苏修梁, 张欣宇. 表面涂层与基体间的界面结合强度及 其测定 $[J]$. 电镀与环保, 2004，24(2)：6-11.

SU Xiuliang, ZHANG Xinyu. Interfacial bonding strength between surface coating and substrate and its measurement[J]. Electroplating \& Pollution Control, 2004, 24(2): 6-11.

[65] 岩下寛之, 森田俊一, 田中厚夫. 鋼板へのラミネート における二軸配向ポリエステルフィルムの無配向層 形成[J]. 鐵と鋼, 2001，87：175-182.

IWASHITA H, MORITA S, TANAKA A. Development of amorphous layer of biaxially oriented polyester film on laminated steel in the laminating process[J]. Tetsu-to-Hagane, 2001, 87(4): 175-182.

[66] 岩下寛之, 森田俊一, 田中厚夫, 等. 鋼板へのラミネ 一トにおける二軸配向ポリエステルフィルムの無配 向層形成におょぼすラミネートロール接触冷却長の 影響 $[J]$. 鉄と鋼, 2001，87(7): 494-499.

IWASHITA H, MORITA S, TANAKA A, et al. Effect of contact cooling length after the laminating rolls on formation of amorphous layer of biaxially oriented polyester film in the laminating process[J]. Tetsu-toHagane, 2001, 87(7): 494-499.

[67] 侯立刚, 时家兴, 汤庆华. 钢板覆膜过程的温度场计算 [J]. 宝钢技术，2015(5): 29-34.

HOU Ligang, SHI Jiaxing, TANG Qinghua. Temperature field calculation for plate laminating process $[\mathrm{J}]$. Baosteel
Technology, 2015(5): 29-34.

[68］宋厚春，王家金. PET 流变曲线校正的研究[J]. 合成技 术及应用，2005，20(1)：54-57.

SONG Houchun, WANG Jiajin. Research on the correction of PET's rheological curves[J]. Synthetic Technology and Application, 2005, 20(1): 54-57.

[69] 谢元彦, 杨海林, 阮建明. 流变学的研究及其应用 [J]. 粉末冶金材料科学与工程, 2010, 15(1): 1-7.

XIE Yuanyan, YANG Hailin, RUAN Jianming. Study and applications of rheology[J]. Materials Science and Engineering of Powder Metallurgy, 2010， 15(1): 1-7.

[70] 孙蓟泉, 尹衍军, 牛闯, 等. 基于流变学理论的 TRIP600 钢本构模型研究 [J]. 机械工程学报, 2016, 52(10): 75-83. SUN Jiquan, YIN Yanjun, NIU Chuang, et al. Research on constitutive model of trip600 based on rheological theory[J]. Journal of Mechanical Engineering, 2016, 52(10): 75-83.

[71] 陈文芳, 蔡扶时. 非牛顿流体的一些本构方程[J]. 力学 学报, 1983, 19(1): 18-28.

CHEN Wenfang, CAI Fushi. Some constitutive equations for non-newtonian fluids[J]. Chinese Journal of Theoretical and Applied Mechanics, 1983, 19(1): 18-28.

[72] 刘海燕, 庞明军, 魏进家. 非牛顿流体研究进展及发展 趋势[J]. 应用化工，2010，39(5): 740-746.

LIU Haiyan, PANG Mingjun, WEI Jinjia. A progress and trend of the non-newtonian fluids[J]. Applied Chemical Industry, 2010, 39(5): 740-746.

[73] 柳和生, 涂志刚, 熊洪槐. 聚合物熔体粘弹性本构方程 [J]. 高分子材料科学与工程, 2002, 18(1): 22-25.

LIU Hesheng, TU Zhigang, XIONG Honghuai. Viscoelastic constitutive equations of polymer melts[J]. Polymer M aterials Science and Engineering, 2002, 18(1): $22-25$.

[74] HAYAT T, ASGHAR S, SIDDIQUI A M. Some unsteady unidirectional flows of a non-Newtonian fluid[J]. International Journal of Engineering Science, 2000, 38(3): 337-345.

[75] 森田俊一, 岩下寬之, 田中厚夫, 等. 鋼板へのラミネ 一トに伴う二軸配向ポリエステルフィルムの配向変 化 $[\mathrm{J}]$. 鐵と鋼, 2000, 86(3): 171-175.

MORITA S, IWASHITA H, TANAKA A, et al. Variations of biaxial orientation of polyester film on laminated steel in the laminating process[J]. Tetsu-to-Hagane, 2000, 86(3): 171-175.

[76] 鈴木威, 岩佐浩樹, 山下正明. PETラミネート鋼板の 湿潤密着機構[J]. 鐵と鋼, 2003，89(1): 142-148. SUZUKI T, IWASA H, YAMASHITA M. The mechanism of wet adhesion of the PET-laminated steel sheet[J]. Tetsu-to-Hagane, 2003, 89(1): 142-148. 
[77] TERAUCHI F, IISHITA H, TANAKA A, et al. Effect of annealing temperature on adhesion of polyester film to electrolytically chromium coated steel[J]. Tetsu-toHagane, 2009, 85(3): 236-240.

[78] CHO C K, KIM J D, CHO K, et al. Effects of the lamination temperature on the properties of poly(ethylene terephthalate-co-isophthalate) in polyester-laminated tin-free steel can - I. Characterization of poly(ethylene terephthalate-co-isophthalate)[J]. Journal of Adhesion Science \& Technology, 2000, 14(9): 1131-1143.

[79] CHO C K, KIM J D, CHO K, et al. Effects of the lamination temperature on the properties of poly(ethylene terephthalate-co-isophthalate) in polyester-laminated tin-free steel can - II. Adhesion mechanism of poly(ethylene terephthalate-co-isophthalate) to TFS[J]. Journal of Adhesion Science \& Technology, 2000, 14(9): 1145-1157.

[80] DING Liping, XIE Long, CAO Jianyun, et al. Crystallization behavior of poly (ethylene terephthalateco-neopentyl terephthalate-co-ethylene isophthalate-coneopentyl isophthalate) copolyester and its application in laminated tin-free steel[J]. Journal of Applied Polymer Science, 2015, 132(31): 42308.

[81] YAMANAKA $\mathrm{Y}$, IWASA $\mathrm{H}$, WATANABE $\mathrm{T}$. Development of laminated tin free steel(TFS) 'UNIVERSAL BRITE (R)' type F for food canst[J]. JFE Technical Report, 2007 (9): 49-53.

[82] BOELEN B, DEN HARTOG H, VAN DER WEIJDE H. Product performance of polymer coated packaging steel, study of the mechanism of defect growth in cans[J]. Progress in Organic Coatings, 2004, 50(1): 40-46.

[83] ZHANG X, BOELEN B, BEENTJES P, et al. Influence of uniaxial deformation on the corrosion performance of pre-coated packaging steel[J]. Progress in Organic Coatings, 2007, 60(4): 335-342.

[84] ZUMELZU E , RULL F , BOETTCHER A A. Characterization and micro- and ultra-structural analysis of PET-based Co-rolled composite electrolytic chromium coated steel (ECCS)[J]. Journal of Materials Processing Tech, 2006, 173(1): 34-39.

[85] ZUMELZU E, CABEZAS C, DELGADO F. Performance and degradation analyses of traditional and ECCS canning tinplates in citric-citrate medium[J]. Journal of Materials Processing Tech, 2004, 152(3): 384-388.

[86] 冯树铭. 聚酯薄膜的质量控制及其性能检测 [J]. 合成 技术及应用，2009，24(1)：44-48.

FENG Shuming. Ployester film quality control and performance testing $[\mathrm{J}]$. Synthetic Technology and Application, 2009, 24(1): 44-48.
[87] 张清东, 黄纶伟, 周晓敏. 宽带钢轧机板形控制技术比 较研究[J]. 北京科技大学学报, 2000, 22(2): 177-181. ZHANG Qingdong, HUANG Lunwei, ZHOU Xiaomin. Comparative study on shape control technologies for wide strip mills $[\mathrm{J}]$. Journal of University of Science and Technology Beijing, 2000, 22(2): 177-181.

[88] 张清东, 张晓峰, 文杰. 薄带钢冷连轧横向厚差控制理 论及 DI 材横向厚差控制技术研究 [J]. 机械工程学报, 2013, 49(24): 30-38.

ZHANG Qingdong, ZHANG Xiaofeng, WEN Jie. Theory and technology of transverse thickness deviation control for di tinplate during tandem cold rolling[J]. Journal of Mechanical Engineering, 2013，49(24): 30-38.

[89] 张清东, 李博, 张晓峰. 带钢平整轧制板形控制行为及 效应的研究 $[J]$. 机械工程学报, 2014, 50(8): 45-52. ZHANG Qingdong, LI Bo, ZHANG Xiaofeng. Research on the behavior and effects of flatness control in strip temper rolling process[J]. Journal of Mechanical Engineering, 2014, 50(8): 45-52.

[90] 张清东, 张勃洋, 李瑞, 等. 镀锡钢板表面光泽度轧制 转印控制[J]. 机械工程学报，2016，52(14)：48-57.

ZHANG Qingdong, ZHANG Boyang, LI Rui, et al. Control of surface glossiness during temper rolling aimed at improving visual aesthetics of tinplate[J]. Journal of Mechanical Engineering, 2016，52(14): 48-57.

[91] 张勃洋, 卢兴福, 张立元, 等. 冷轧极薄带钢复杂板形 翅曲变形行为研究 [J]. 机械工程学报，2018，54(12): 184-192.

ZHANG Boyang, LU Xingfu, ZHANG Liyuan, et al. Analysis of complex warping deformation for cold-rolled strip $[\mathrm{J}]$. Journal of Mechanical Engineering, 2018, 54(12): 184-192.

[92] 张清东, 张勃洋, 李瑞, 等. 钢板微观表面质量控制理 论与技术研究进展 $[\mathrm{J}]$. 机械工程学报, 2016, 52(10): 32-45.

ZHANG Qingdong, ZHANG Boyang, LI Rui, et al. Advances in theory and technology for microscopic surface quality control of steel strip[J]. Journal of Mechanical Engineering, 2016，52(10): 32-45.

[93] 高宏保. 国内聚酯薄膜现状及发展趋势 [J]. 合成技术 及应用，2006，21(4)：25-28.

GAO Hongbao. Requirment of raw PET pellets for high-modulus and low-shrinkage commercial filaments[J]. Synthetic Technology and Application, 2006, 21(4): 25-28.

[94] 高枫. 国外 PET 聚酯技术现状与进展 [J]. 国外塑料, 2010, 28(8): 54-57.

GAO Feng. Status and progress of foreign PET polyester technology[J]. World Plastics, 2010, 28(8): 54-57. 
[95] BAHLAKEH G, RAMEZANZADEH B, SAEB, MR. Corrosion protection properties and interfacial adhesion mechanism of an epoxy/polyamide coating applied on the steel surface decorated with cerium oxide nanofilm: Complementary experimental, molecular dynamics (MD) and first principle quantum mechanics (QM) simulation methods[J]. Applied Surface Science, 2017, 419: 650-669.

[96] RANJBAR N, TALEBIAN S, MEHRALI M, et al. Mechanisms of interfacial bond in steel and polypropylene fiber reinforced geopolymer composites[J]. Composites Science \& Technology, 2016, 122: 73-81.

[97] WU Y, WANG A H, ZHENG R R, et al. Laser-drilled micro-hole arrays on polyurethane synthetic leather for improvement of water vapor permeability[J]. Applied Surface Science, 2014, 305(7): 1-8.

[98] ZHANG Xi, MA Quanyang, DAI Yu, et al. Effects of surface treatments and bonding types on the interfacial behavior of fiber metal laminate based on magnesium alloy[J]. Applied Surface Science， 2017， 427: 897-906.

[99] GAO Chengcheng, BAO Xianyang, YU Long, et al. Thermal properties and miscibility of semi-crystalline and amorphous PLA blends[J]. Journal of Applied Polymer Science, 2014, 131(23): 41205.

[100] MAHDAOUI M, KOUSKSOU T, MARÍN J M, et al. Numerical simulation for predicting DSC crystallization curves of tetradecane-hexadecane paraffin mixtures[J]. Thermochimica Acta, 2014, 591: 101-110.

[101] THAKRAL S, TERBAN M W, THAKRAL N K, et al. Recent advances in the characterization of amorphous pharmaceuticals by X-ray diffractometry[J]. Advanced Drug Delivery Reviews， 2016，100: 183-193.

[102] ARNDT U W, BLOOMER A C. New developments in $\mathrm{X}$-ray optics for macromolecular crystallography using laboratory X-ray sources[J]. Current Opinion in Structural Biology, 1999, 9(5): 609-614.

[103] 沈新元. 高分子材料与工程专业实验教程[M]. 北京: 中国纺织出版社, 2010.

SHEN Xinyuan. Polymer materials and engineering experiment[M]. Beijing: China Textile \& Apparel Press, 2010.

[104] 李承花, 张奕, 左琴华, 等. 差式扫描量热仪的原理 与应用 $[J]$. 分析仪器, 2015 (4): 88-94.

LI Chenghua, ZHANG Yi, ZUO Qinhua, et al. Application of differential scanning calorimeter[J]. Analytical Instrumentation, 2015 (4): 88-94.

[105] 丁丽萍. 覆膜铁用功能聚酯的合成及其应用研究 [D]. 哈尔滨：哈尔滨工业大学, 2015.

DING Liping. Synthesis and application of functional polyester for laminated tin-free steel[D]. Harbin: Harbin
Institute of Technology，2015.

[106] 凌绳, 王秀芬, 吴友平. 聚合物材料[M]. 北京: 中国 轻工业出版社, 2009.

LING Sheng, WANG Xiufen, WU Youping. Ploymer materials[M]. Beijing: China Light Industry Press, 2009.

[107] JABARIN S A. Crystallization kinetics of poly (ethylene terephthalate). III. Effect of moisture on the crystallization behavior of PET from the glassy state[J]. Journal of Applied Polymer Science, 1987, 34(1): 103-108.

[108] DEMIREL B， YARAŞ A， ELÇIÇEK H. Crystallization behavior of PET materials[J]. Balıkesir Üniversitesi Fen Bilimleri Enstitüsü Dergisi， 2016， 13(1): 26-35.

[109] 陈俊, 陈剑玲, 刘正英, 等. PET 结晶行为研究进展 [J]. 高分子通报，2005(1): 20-24.

CHEN Jun, CHEN Jianling, LIU Zhengying, et al. Advances in crystallization behavior of polyethylene terephthalate[J]. Chinese Polymer Bulletin, 2005(1): 20-24.

[110] 严大东, 张兴华. 聚合物结晶理论进展[J]. 物理学报, 2016, 65(18): 101-111.

YAN Dadong, ZHANG Xinghua. Recent development on the theory of polymer crystallization[J]. Acta Physica Sinica, 2016, 65(18): 101-111.

[111] 边界, 叶胜荣, 封麟先. PET 类共聚酯的玻璃化转变 $[\mathrm{J}]$. 高等学校化学学报, 2003, 24(1): 155-159.

BIAN Jie, YE Shengrong, FENG Linxian. The glass transit ion of copolyester[J]. Chemical Journal of Chinese Universities, 2003, 24(1): 155-159.

[112] VAN DER AA M A H, SCHREURS P J G, BAAIJENS F P T. Modelling of the wall ironing process of polymer coated sheet metal[J]. Mechanics of Materials, 2001, 33(10): 555-572.

[113] VAN TIJUM R, VELLINGA W P, DE HOSSON J T M. Surface roughening of metal-polymer systems during plastic deformation[J]. Acta Materialia, 2007, 55(8): 2757-2764.

[114] VAN DER AA H C E, VAN DER AA M A H, SCHREURS P J G, et al. An experimental and numerical study of the wall ironing process of polymer coated sheet metal[J]. Mechanics of Materials, 2000， 32(7): 423-443.

[115] VAN BEECK J, MARESCA F, DE GEUS T W J, et al. Predicting deformation-induced polymer-steel interface roughening and failure[J]. European Journal of Mechanics-A/Solids, 2016, 55: 1-11.

[116] 曹贵邦. 覆膜铁制罐拉深成形过程缺陷及其机理研究 [D]. 广州: 广东工业大学, 2017.

CAO Guibang. Study on the defects and their 
mechanism in the deep-drawing process for the polymer laminated steel can forming[D]. Guangzhou: Guangdong University of Technology, 2017.

[117] 江轲, 程柏松, 屈战龙, 等. 冲压成形前热处理对覆 膜铁制罐白痕的影响[J]. 中国包装工业, 2014(8): 4-5. JIANG Ke, CHENG Bosong, QU Zhanlong, et al. Effect of heat treatment before stamping on white marks of coated iron cans[J]. China Packaging Industry, 2014(8): 4-5.

[118] 倪俊义, 江辑, 张作全. 烘烤工艺对彩印覆膜铁 DRD 罐性能的影响 $[\mathrm{J}]$. 包装工程, 2014(17): 69-71.

NI Junyi, JIANG Ke, ZHANG Zuoquan. Effects of baking process on performance of printed laminated steel DRD cans[J]. Packaging Engineering, 2014(17): 69-71.

[119] 倪俊义, 张作全, 黄晶, 等. 覆膜铁在食品 DRD 罐上 的应用[J]. 功能材料, 2012, 43(s2): 308-310.

NI Junyi, ZHANG Zuoquan, HUANG Jing, et al. The use of laminated steel on food packaging[J].Journal of Function Materials, 2012, 43(s2): 308-310.

[120] MOUSA S, SCHEIRER N， KIM G Y. Roll-bonding of metal-polymer-metal sandwich composites reinforced by glass whiskers at the interface[J]. Journal of Materials Processing Technology, 2018, 255: 463-469.

[121] BEECK J V, BREEMEN L C A V, SCHREURS P J G, et al. Preventing interface damage by pre-conditioning polymer-coated steels via rolling[J]. International Journal of Solids \& Structures, 2015, 58: 1-11.

[122] OCHOA-PUTMAN C, VAIDYA U K. Mechanisms of interfacial adhesion in metal polymer composites-Effect of chemical treatment[J]. Composites Part A Applied Science \& Manufacturing, 2011, 42(8): 906-915.

[123] LINDNER M, RODLER N, JESDINSZKI M, et al. Surface energy of corona treated PP, PE and PET films, its alteration as function of storage time and the effect of various corona dosages on their bond strength after lamination[J]. Journal of Applied Polymer Science, 2018, 135(11): 45842(1-9).

[124] YANG Liqing, CHEN Jierong, GUO Yafei, et al. Surface modification of a biomedical polyethylene terephthalate (PET) by air plasma[J]. Applied Surface Science, 2009, 255(8): 4446-4451.

[125] PASCOE R D, O'CONNELL B. Flame treatment for the selective wetting and separation of PVC and PET[J]. Iste Management, 2003, 23(9): 845-50.

[126] JIANG Zhongqing, JIANG Zhongjie. Plasma techniques for the fabrication of polymer electrolyte membranes for fuel cells[J]. Journal of Membrane Science, 2014, 456(15): 85-106.
[127] KUMAR V，SONKAWADE R G，CHAKARVARTI S

$\mathrm{K}$, et al. Carbon ion beam induced modifications of optical, structural and chemical properties in PADC and PET polymers $[\mathrm{J}]$. Radiation Physics \& Chemistry, 2012, 81(6): 652-658.

[128] PANDIYARAJ K N, SELVARAJAN V, DESHMUKH R $\mathrm{R}$, et al. Adhesive properties of polypropylene (PP) and polyethylene terephthalate (PET) film surfaces treated by DC glow discharge plasma[J]. Vacuum, 2008, 83(2): 332-339.

[129] MOHAMED R, DAVID B, STÉPHANE M, et al. Impact of corona treated hemp fibres onto mechanical properties of polypropylene composites made thereof $[\mathrm{J}]$. Industrial Crops \& Products, 2010， 31(2): 344-349.

[130] LAHTI J, SAVOLAINEN A, RÄSÄNEN J P, et al. The role of surface modification in digital printing on polymer-coated packaging boards[J]. Polymer Engineering \& Science, 2004, 44(11): 2052-2060.

[131] FÖLDES E, TÓTH A, KÁLMÁN E, et al. Surface changes of corona-discharge-treated polyethylene films[J]. Journal of Applied Polymer Science, 2015, 76(10): 1529-1541.

[132] DING Liping, WANG Lipeng, SHAO Lu, et al. The water-dependent decay mechanism of biaxially-oriented corona-treated polyethylene terephthalate films[J]. Rsc Advances, 2014, 4(97): 54805-54809.

[133] CARRADÒ A, SOKOLOVA O, DONNIO B, et al. Influence of corona treatment on adhesion and mechanical properties in metal/polymer/metal systems[J]. Journal of Applied Polymer Science, 2015, 120(6): 3709-3715.

[134] BARBARO G, GALDI M R, MAIO L D, et al. Effect of BOPET film surface treatments on adhesion performance of biodegradable coatings for packaging applications[J]. European Polymer Journal, 2015, 68: 80-89.

[135] KALAPAT N, AMORNSAKCHAI T, SRIKHIRIN T. Surface modification of biaxially oriented polypropylene (BOPP) film using acrylic acid-corona treatment: Part II. Long term aging surface properties[J]. Surface \& Coatings Technology, 2013, 234(10): 67-75.

[136] SANTOS L P, BERNARDES J S, GALEMBECK F. Corona-treated polyethylene films are macroscopic charge bilayers[J]. Langmuir, 2013， 29(3): 892.

[137] RAGOUBI M, GEORGE B, MOLINA S, et al. Effect of corona discharge treatment on mechanical and thermal properties of composites based on miscanthus fibres and polylactic acid or polypropylene matrix $[\mathrm{J}]$. Composites Part A, 2012, 43(4): 675-685. 
[138] HAN M H, JEGAL J P, PARK K W, et al. Surface modification for adhesion enhancement of PET-laminated steel using atmospheric pressure plasma[J]. Surface \& Coatings Technology, 2007, 201(9-11): 4948-4952.

[139] PELAGADE S M, SINGH N L, QURESHI A, et al. Investigation of surface properties of Ar-plasma treated polyethylene terephthalate (PET) films[J]. Nuclear Inst \& Methods in Physics Research B, 2012, 289(11): 34-38.

[140] 邓娟, 高燕, 张波. 废旧 PET 共混改性研究进展 [J]. 塑 料助剂, 2018, 128(2): 10-12.

DENG Juan, GAO Yan, ZHANG Bo. Research progress on the blending modification of the waste PET[J]. Plastics Additives, 2018, 128(2): 10-12.

[141] 史君. PET 合成与改性新工艺及其合金制备研究 [D].
北京: 北京化工大学, 2009.

SHI Jun. Study on new process of synthesis and modification for PET and preparation of its alloy materials[D]. Beijing: Beijing University of Chemical Technology, 2009.

[142] 王志远, 陈福林, 张兴华. 国内 PET 改性研究的最新 进展 $[J]$. 聚酯工业, 2006, 19(3): 1-4.

WANG Zhiyuan, CHEN Fulin, ZHANG Xinghua. The domestic latest research advance of PET modification[J]. Ployester Industry, 2006, 19(3): 1-4.

作者简介: 张清东, 男, 1965 年出生, 博士, 教授, 博士研究生导师。 主要研究方向为板带轧制与板形厚度表面控制, 塑性成形装备与工艺界 面力学行为, 薄带屈曲与皱曲。

E-mail: zhang_qd@me.ustb.edu.cn

刘吉阳(通信作者), 男, 1992 年出生, 博士研究生。主要研究方向为覆 膜铁覆膜机理与关键技术。

E-mail: b20170270@xs.ustb.edu.cn

\section{第 8 届上银优秀机械博士论文奖一一佳作奖}

\section{煤化工严苛工况阀门多相流冲蚀磨损一气蚀机理及预测方法研究}

作 者: 郑智剑

毕业学校: 浙江理工大学

指导教师: 偶国富

本文以煤化工严苛工况划门内多相流冲蚀磨损-气蚀失效为研究对象, 明确了复杂流动条件下阀内件的失效机理及损伤 过程，并建立含气液相变的多相流冲蚀磨损一气蚀预测方法。通过工艺过程、运行状态分析、受损表面微观形貌测试，基本 明确了热高分液控阀和高压黑水角阀的失效机理; 通过阀门空化-空蚀试验和高温冲蚀磨损试验, 研究阀门气蚀和冲蚀磨损 机理, 并对空化模型和颗粒冲蚀磨损模型进行修正; 构建了含气液相变的多相流冲蚀磨损-气蚀数学模型, 并提出阀内流动 核心区域、冲蚀磨损和气蚀的表征参数; 在验证数学模型和计算方法准确性的基础上，分别开展热高分液控阀和高压黑水角 阀的冲蚀磨损-气蚀预测研究，获得了阀门在不同运行阶段的失效机理。与失效案例进行对比，验证了计算结果的可靠性; 提出并验证了阀门流道结构的改进方案。

本文的创新性工作在于: 利用阀门空化流动试验数据和高温冲蚀磨损试验数据, 对空化模型和冲蚀磨损模型进行修正, 并验证了计算模型和方法的准确性; 研究阀芯结构的改变对冲蚀磨损-气蚀规律的影响, 获得阀门在不同运行阶段的失效机 理, 提出并验证了阀芯及流道结构的改进方案; 采用凝结速率表征阀内的空蚀损伤, 结合空蚀试验数据, 验证了表征参数的 准确性。

本文的工程应用价值在于：建立的含气液相变的多相流冲蚀磨损-气蚀数学模型和表征参数，提高了复杂流动条件下阀 门失效预测的准确性; 建立的数值计算方法, 能对阀门的设计方案进行流动腐蚀预测和校核, 降低制造和研发成本; 在失效 过程分析的基础上, 提出了阀门流道结构及操作的改进方案, 有效延长了阀门的使用寿命, 减少了非计划停工次数, 经济效 益显著。同时，推动了重大阀门装备设计、制造的国产化进程。 